\title{
Médiévales
}

Langues, Textes, Histoire

69 | automne 2015

Travailler à Paris (XIIIe-XVIe siècle)

\section{Le travail au Moyen Âge, à Paris et ailleurs : retour sur l'histoire d'un modèle}

Labour in the Middle Ages, in Paris and elsewhere : history of a model

\section{Christine Jéhanno}

\section{(2) OpenEdition \\ 12 Journals}

\section{Édition électronique}

URL : https://journals.openedition.org/medievales/7567

DOI : 10.4000/medievales.7567

ISSN : 1777-5892

Éditeur

Presses universitaires de Vincennes

\section{Édition imprimée}

Date de publication : 30 novembre 2015

Pagination : 5-17

ISBN : 978-2-84292-444-7

ISSN : 0751-2708

\section{Référence électronique}

Christine Jéhanno, "Le travail au Moyen Âge, à Paris et ailleurs : retour sur l'histoire d'un modèle ", Médiévales [En ligne], 69 | automne 2015, mis en ligne le 30 novembre 2017, consulté le 08 décembre 2022. URL : http://journals.openedition.org/medievales/7567; DOI : https://doi.org/10.4000/ medievales.7567 
Christine Jéhanno

\section{Le travail au Moyen Âge, à Paris et ailleurs : retour sur l'histoire d'un modèle}

Si Jacques Le Goff, récemment disparu, affirmait que « le travail n'est pas un bon sujet pour le Moyen Âge ${ }^{1}$ ", c'était à l'évidence, de la part de ce spécialiste, pour mettre en garde contre la difficulté à saisir une réalité médiévale qui n'avait pas de mot pour se dire et non pour dissuader de s'y intéresser ${ }^{2}$. De fait, son avertissement n'a pas découragé les médiévistes, loin s'en faut. En un temps de crise économique et de chômage, la question du travail n'a du reste pas manqué d'être investie par l'ensemble des sciences humaines. Les historiens étaient ainsi appelés, en 2001, à apporter leur contribution à l'effort de réflexion commun dans un dossier des Cahiers d'Histoire. Revue d'histoire critique qui titrait «Comment les historiens parlent-ils du travail ? », dans une perspective trans-périodique. Catherine Verna et Philippe Bernardi y répondaient pour les médiévistes dans un article intitulé « Travail et Moyen-Âge : un renouveau historiographique », dans lequel ils dressaient non seulement un bilan, mais indiquaient aussi les pistes alors dégagées par la recherche historique en la matière, et concluaient à une « effervescence ${ }^{3} »$.

1. J. LE GoFF, « Discours de clôture », dans Le Travail au Moyen Âge. Une approche interdisciplinaire, Actes du colloque international de Louvain-la-Neuve, mai 1987, Louvainla-Neuve, 1990, p. 424.

2. Ce propos reprenait l'idée déjà formulée au colloque de Spolète en 1970 par J. LE GoFF, « Travail, techniques et artisans dans les systèmes de valeur du haut Moyen Âge ( $V^{\mathrm{e}}-\mathrm{X}^{\mathrm{e}}$ siècles) ", dans Artigianato e Tecnica nella Società dell'Alto medioevo occidentale(2-8 aprile 1970), Spolète, 1971, p. 240. L'historien reconnaissait lui-même à Louvain en 1987 que son propos d'alors avait été dit «d'une façon un peu provocante » (J. LE GoFF, « Discours de clôture », dans Le Travail au Moyen Âge..., p. 414).

3. C. VERNA et P. BERNARDI, «Travail et Moyen Âge: un renouveau historiographique », dans Comment les historiens parlent-ils du travail ?, Cahiers d'Histoire. Revue d'histoire critique, 83 (2001), p. 27-46. Catherine Saliou et Philippe Minard répondaient eux du point de vue de l'antiquisant et du moderniste, respectivement p. 13-26 et 47-66. 
Il y a peu, étaient ainsi menés plusieurs projets autour du sujet. Parmi ceux-ci, le programme «Salaire et salariat au Moyen Âge ( $\mathrm{XI}^{\mathrm{e}}-\mathrm{XVI}^{\mathrm{e}} \mathrm{s}$.) », coordonné par Laurent Feller de 2006 à 2008 ${ }^{4}$, s'est intéressé aux rapports de travail dans l'ensemble de l'Europe médiévale. La question était ensuite largement abordée en 2011-2013 dans le cadre du séminaire de François Menant à l'ENS-Ulm consacré aux milieux populaires urbains de la fin du Moyen Âge ${ }^{5}$, puis lors du colloque «Clivages sociaux et modes de domination dans les villes européennes des $\mathrm{XIII}^{\mathrm{e}}-\mathrm{XV}^{\mathrm{e}}$ siècles $\gg$ tenu à Paris les 20 et 21 juin 2013. Parallèlement, à l'Université de Paris 8-Saint-Denis, Catherine Verna choisissait pour thème de son séminaire de recherche « Le travail au Moyen Âge : techniques, production, marchés ». C'est dans cette convergence que le groupe de travail sur Paris au Moyen Âge de l'IRHT a souhaité s'inscrire. Il semblait en effet important à ses membres d'y insérer Paris, dont le cas, longtemps sur le devant de la scène en ce domaine, puis quelque peu délaissé, méritait de trouver place ${ }^{6}$. Ils sont heureux que la revue Médiévales ait accepté la publication de quelques-unes des interventions, parmi celles consacrées à Paris et ses environs, extraites de la trentaine d'un séminaire poursuivi de 2012 à 2014, qui avait volontairement élargi ses investigations à d'autres espaces, afin de multiplier les points de comparaison, tout en restant centré sur le travail urbain pris dans sa dimension productive.

Même ainsi circonscrit, le champ d'étude affiche un bilan historiographique des plus riches et chaque nouvelle publication s'ouvre sur une remarque relative à l'abondance de la bibliographie ${ }^{7}$, fournissant une liste de références ou, plus souvent encore, renvoyant faute de place à une synthèse, en général ancienne, ou à un bilan historiographique récent ${ }^{8}$.

4. Le programme a donné lieu à une publication : P. BECK, P. BERNARDI et L. FELLER éd., Rémunérer le travail au Moyen Âge : pour une histoire sociale du salariat, Paris, 2014.

5. Dans le cadre du séminaire tenu de 2010 à 2013 par François Menant et Diane Chamboduc de Saint-Pulgent intitulé « Les sociétés européennes au Moyen Âge : modèles d'interprétation, pratiques, langages », qui a porté en 2011-2013 sur les groupes populaires urbains des $\mathrm{XIII}{ }^{\mathrm{e}}-\mathrm{XV}^{\mathrm{e}}$ siècles.

6. Convergences attestées par le fait que les participants aux divers séminaires ou programmes ont été invités à s'exprimer dans les autres.

7. Même en s'en tenant à la littérature scientifique, c'est-à-dire en excluant les innombrables ouvrages de vulgarisation. Il n'a malheureusement pas été possible de prendre en considération les mémoires de master traitant du sujet, pourtant nombreux, et qui attestent aussi à leur manière sa vitalité.

8. Parmi ceux-ci, on lira avec grand profit, outre l'article déjà cité de Catherine Verna et Philippe Bernardi («Travail et Moyen Âge... »), la première partie de P. BERNARDI, Maître, valet et apprenti : essai sur une production bien ordonnée, Toulouse, 2009, p. 23-83. Sur la question particulière du salariat, un bilan historiographique a été fait en mai 2006, lors de la rencontre d'Avignon qui allait lancer le programme « Salaire et salariat au Moyen Âge $\left(\mathrm{XI}^{\mathrm{e}}-\mathrm{XVI}^{\mathrm{e}}\right.$ siècle) » (textes des communications consultables en ligne sur le site du LaMOP : http://lamop.univ-paris1.fr/IMG/pdf/Salaire_salariat_1.pdf). Dans une perspective étendue 
La situation n'est pas propre au Moyen Âge car le champ de recherche est exploré pour toutes les périodes historiques et, à l'intérieur de chacune d'elles, pour l'ensemble des siècles ${ }^{9}$. Du reste, nombre d'ouvrages s'affranchissent des barrières chronologiques académiques, ambitionnant pour certains une histoire au long cours « des origines à nos jours », englobant en tout cas couramment la période des $\mathrm{XIII}^{\mathrm{e}}-\mathrm{XVI}^{\mathrm{e}}$ siècles, voire Moyen Âge et Temps modernes ${ }^{10}$. Le propos n'est pas dans cette introduction de faire à nouveau l'historiographie du travail mais, en la brossant à gros traits, de pointer la place singulière que tient le Paris du Moyen Âge dans une recherche commencée dès le XIX ${ }^{\mathrm{e}}$ siècle, et qui s'est poursuivie sans réelle interruption depuis, même à bas bruit et même si le sujet occupe rarement le devant de la scène ${ }^{11}$.

Les bases de l'étude du travail au Moyen Âge sont posées en 1840 , avec l'Essai sur l'état des corporations industrielles au Moyen-Âge d'Henri de Formeville. Le XIX ${ }^{\mathrm{e}}$ siècle et le début du $\mathrm{XX}^{\mathrm{e}}$ siècle sont, toutes périodes historiques confondues, un moment d'intense production sur le sujet puisque, en 1885, Hippolyte Blanc juge bon de publier une Bibliographie des corporations ouvrières ${ }^{12}$, dans laquelle il recense déjà près de mille titres. Parmi ceux-ci figurent quelques amples vues d'ensemble comme, en 1859, l'Histoire des classes ouvrières en France de Jules César à la Révolution d'Émile Levasseur, alors qu'en 1899, Henri Hauser s'en tient à un cadre chronologique plus restreint dans Ouvriers du temps passé ( $X V^{e}$ $X V I^{e}$ siècle). Au début du $\mathrm{XX}^{\mathrm{e}}$ siècle vient le temps des premières grandes synthèses, notamment l'Histoire universelle du travail, sous la direction de Georges Renard, dont le tome consacré au Moyen Âge, Le Travail dans l'Europe chrétienne au Moyen Âge : $V^{e}-X V^{e}$ siècle, paraît en 1921 sous la plume de Prosper Boissonnade. Or les ouvrages de ce temps, bien que censés rendre compte d'une réalité générale, sont largement inspirés de l'exemple parisien. À cela, une raison simple : les sources parisiennes de l'histoire du travail ont été parmi les premières éditées. Le Livre des métiers d'Étienne Boileau est publié d'abord par Georges-Bernard Depping dès

et pour la période récente : P. Bernardi, M. ARnouX et P. Braunstein, « Production, travail, consommation », dans J.-C. SCHMITT et O. G. OEXLE éd., Les Tendances actuelles de l'histoire du Moyen Âge en France et en Allemagne, Paris, 2002.

9. Compte tenu du champ couvert par le séminaire d'histoire de Paris au Moyen Âge de l'IRHT, le haut Moyen Âge n'a pas été pris en compte ici.

10. Les années 1250-1650 pour M. ARNOUX et P. MONNET éd., Le Technicien dans la cité en Europe occidentale, 1250-1650, Rome, 2004 ; les $\mathrm{V}^{\mathrm{e}}-\mathrm{XVIII}{ }^{\mathrm{e}}$ siècles pour le deuxième tome de l'Histoire du travail : P. WOLFF et F. MAURO, L'Âge de l'artisanat (V'e-XVIII'siècles), Paris, 1959.

11. Dès lors, il a été jugé pertinent de privilégier ici l'historiographie française.

12. H. BlanC, Bibliographie des corporations ouvrières, Paris, 1885. 
$1837^{13}$, puis par François Bonnardot et René de Lespinasse en 1879, avec une solide introduction ${ }^{14}$. C'est ensuite le tour des ordonnances rassemblées en trois tomes par René de Lespinasse ${ }^{15}$, puis des Livres de couleur et des Livres de bannières du Châtelet de Paris par Alexandre Tuetey au tournant $\mathrm{du} \mathrm{XX}^{\mathrm{e}}$ siècle ${ }^{16}$. Le corpus mis ainsi à disposition est considérable et semble à même de révéler la situation parisienne. Après une série d'articles entre 1868 et $1874{ }^{17}$, Gustave Fagniez présente la synthèse de ses travaux sous le titre Études sur l'industrie et la classe industrielle à Paris, au XIII et au XIV siècle, en $1877^{18}$. Le titre choisi témoigne de la largeur de vue de l'ouvrage. Mais, compte tenu des sources parisiennes, il est malgré tout axé sur les métiers, tant le métier - on emploie alors plutôt le terme de « corporation »- constitue le centre d'intérêt premier, la voie d'accès privilégiée à l'histoire du travail. Dans la décennie suivante, Alfred Franklin publie ainsi Les Corporations ouvrières de Paris, $d u$ XII ${ }^{e}$ au XVIII siècle, puis plusieurs livraisons de la collection La Vie privée d'autrefois, consacrées aux métiers en général ou à certains d'entre eux ${ }^{19}$, et enfin, en point d'orgue, au tout début $\mathrm{du} \mathrm{XX}^{\mathrm{e}}$ siècle, son Dictionnaire historique des arts, métiers et professions exercés à Paris depuis le XIII siècle jusqu'au XVIII ${ }^{e}$ siècle ${ }^{20}$.

13. G.-B. DEPPING, Règlements sur les arts et métiers de Paris, rédigés au XIIT siècle et connus sous le nom de Livre des métiers d'Étienne Boileau, Paris, 1837. Le sous-titre proclame : Publiés pour la première fois en entier d'après les manuscrits de la bibliothèque du roi et des archives du royaume, avec des notes et une introduction.

14. R. DE LESPINASSE et F. BONNARDOT, Les Métiers et les corporations de la ville de Paris au XIII siècle : le Livre des métiers d'Étienne Boileau, Paris, 1879.

15. R. DE LESPINASSE, Les Métiers et corporations de la ville de Paris, Paris, t. I : XIVeXVIII siècles. Ordonnances générales, métiers de l'alimentation, 1886, t. II : XIVe-XVIII siècles. Orfèvrerie, sculpture, mercerie, ouvriers en métaux, bâtiment et ameublement, 1892, t. III : XIV'-XVIII siècles. Tissus, étoffes, vêtements, cuirs et peaux, métiers divers, 1897. D'autres ordonnances sont éditées ponctuellement ensuite, notamment J.-M. RICHARD, « Ordonnance inédite de Philippe le Bel concernant les métiers de Paris (7 juillet 1307) », Mémoires de la Société de l'histoire de Paris et de l'Île-de-France, 2 (1875), p. 130-141.

16. A. TUETEY, Inventaire analytique des livres de couleur et bannières $d u$ Châtelet de Paris, Paris, 1899-1907.

17. G. FAGNIEZ, «Essai sur l'organisation de l'industrie à Paris aux XIII ${ }^{\mathrm{e}}$ et XIV ${ }^{\mathrm{e}}$ siècles », Bibliothèque de l'École des chartes, 29 (1868), p. 1-32 ; 30 (1869), p. 80-105 ; 35 (1874), p. 478-542.

18. G. FAGNIEZ, Études sur l'industrie et la classe industrielle à Paris, au XIII et au XIV siècle, Paris, 1877.

19. A. Franklin, Les Corporations ouvrières de Paris, du XII au XVIII siècle : histoire, statuts, armoiries, d'après des documents originaux ou inédits, Paris, 1885 ; ID., La Vie privée d'autrefois. Arts et métiers, modes, mœurs, usages des Parisiens du XII au XVIII siècle d'après des documents originaux ou inédits. Comment on devenait patron, Paris, 1889 ; ID., Les Médicaments, Paris, 1891 (sur les apothicaires, épiciers et herbiers avec le texte complet des Statuts des apothicaires et épiciers de Paris de 1484, p. 241-254) ; ID., Les Médecins, Paris, 1892 ; ID., Les Chirurgiens, Paris, 1893.

20. A. FRANKLIN, Dictionnaire historique des arts, métiers et professions exercés à Paris depuis le XIII siècle jusqu'au XVIII e siècle, Paris, 1905-1906. 
D'autres études sont menées sur certains métiers ou groupes de métiers parisiens tels les ménestriers, les boulangers, les tailleurs, les bouchers et les artisans du textile ${ }^{21}$.

Dans les ouvrages généraux, de façon explicite ou non, Paris est érigé en modèle à partir duquel est extrapolée la situation du royaume tout entier. En 1941, Émile Coornaert dresse un vaste panorama des Corporations en France avant 1789 après s'être intéressé à celles de Paris à partir du Livre des métiers ${ }^{22}$. Il est du reste conscient de ce que cette démarche peut avoir de simplificateur et appelle d'autres à corriger dès que possible sa « vue cavalière ».

De manière générale, l'accent est mis sur les formes d'organisation professionnelle, les structures. Influencés par la situation documentaire parisienne, les historiens du travail se tournent pour l'essentiel vers des textes normatifs, facilement accessibles et qui alors paraissent transparents. Cela conduit à faire du travail une histoire très institutionnelle. La recherche est fortement marquée en outre par une grille de lecture juridique, qu'elle soit ou non le fait de juristes, notamment de chartistes, très imprégnés alors $\mathrm{d}^{\prime}$ histoire des institutions ${ }^{23}$. De cette époque date la distinction, qui va devenir traditionnelle, entre métiers jurés (s'organisant eux-mêmes, sur le modèle parisien) et métiers réglés (organisés par les pouvoirs publics, à l'exemple de la Flandre ou du Languedoc). Le recours à des sources normatives conduit par ailleurs à construire une vision idéalisée des corporations. Ainsi les historiens du XIX ${ }^{\mathrm{e}}$ siècle créent-ils à partir des métiers, montrés par les statuts comme modèles d'équilibre et éléments de stabilité, la fiction d'une concorde sociale, très recherchée dans un monde industriel alors en plein bouleversement du fait des formes nouvelles prises par le capitalisme mais aussi des revendications ouvrières. Cette idée rencontre du reste celle du catholicisme social qui émerge au moment même de la redécouverte du

21. B. BERNARD, « Recherches sur l'histoire de la corporation des ménestriers et joueurs d'instruments de la ville de Paris », Bibliothèque de l'École des chartes, 3 (1841-1842), p. 377-404 ; 4 (1842-1843), p. 525-548 ; 5 (1843-1844), p. 254-284 et 339-372 ; P. HiVONNAIT, Histoire de la corporation des anciens talemeliers à Paris, du XIII au XVIII siècle, Paris, 1910 ; P. VIDAL, Histoire de la corporation des tailleurs d'habits, pourpointiers, chaussetiers de la ville de Paris, d'après des documents originaux ou inédits, Paris, 1923 ; R. HÉRON DE VILlEFOSSE, « La Grande Boucherie de Paris », Bulletin de la Société de l'histoire de Paris et de l'Île-de-France, 55ªnnée, 1928 (1929), p. 39-73 ; R. GOURMELON, « L'industrie et le commerce des draps à Paris du XII ${ }^{\mathrm{e}}$ au XVI ${ }^{\mathrm{e}}$ siècle », dans Positions des thèses de l'École des chartes, 1950, p. 61-63 ; H. NoCQ. Le Poinçon de Paris. Répertoire des mâ̂tres-orfèvres de la juridiction de Paris depuis le Moyen Âge jusqu'à la fin du XVIII siècle, Paris, 1926-1931.

22. E. CoORnAERT, Les Corporations en France avant 1789, Paris, 1941 ; ID., « Notes sur les corporations parisiennes au temps de saint Louis, d'après le Livre des métiers d'É. Boileau », Revue historique, 177 (1936), p. 343-352.

23. Par exemple : P. VIOLLET, «Les corporations au Moyen Âge », Nouvelle revue historique de droit français et étranger, 24 (1900), p. 624-656. 
Moyen Âge, où l'on va puiser un modèle d'organisation de la production, idéalisé, que Paris est à même de fournir.

Cette instrumentalisation du travail médiéval se reproduit avec l'instauration du corporatisme comme doctrine d'État par les régimes d'inspiration fasciste en Europe. L'historiographie des années 1930 et 1940 se cristallise autour de ce problème ${ }^{24}$. La fin de la guerre qui emporte la plupart de ces régimes, et en France le régime de Vichy, signe le discrédit du corporatisme et oblige des auteurs, qui s'en étaient fait les chantres, à réviser leur point de vue de façon significative. Ainsi Maurice BouvierAjam, qui a publié en 1941 La Doctrine corporative, écrit en 1957 une Histoire du travail en France des origines à la Révolution. L'Histoire du travail d'Édouard Dolléans, sortie en 1944, est totalement revue par le nouveau co-auteur Gérard Dehove et reparaît en 1953 sous le titre Histoire du travail en France. Mouvement ouvrier et législation sociale, qui en dit long sur le tournant pris. Dans les décennies qui suivent, une approche nouvelle de la question du travail se fait jour, désormais dominée par la question du mouvement ouvrier et de la lutte des classes ${ }^{25}$. Le travailleur y occupe une place plus centrale, mais est surtout vu comme membre d'un collectif ${ }^{26}$. Le Moyen Âge n'y trouve plus la place éminente d'autrefois, sauf envisagé au prisme des révoltes, pas spécifiquement urbaines au demeurant, par Philippe Wolff ${ }^{27}$. Paris n' apparaît plus guère au premier plan dans les ouvrages généraux, et les recherches sur l'artisanat de la capitale se réduisent. Pourtant, l'ouvrage de Bronislaw Geremek sur le salariat de l'artisanat parisien, rapidement traduit en plusieurs langues, replace Paris dans la lumière. Son retentissement est considérable tant il vieillit les ouvrages antérieurs, renouvelle les problématiques, et ce, bien au-delà de l'histoire du travail parisien ${ }^{28}$.

24. En France par exemple, F. OLIVIER-MARTIN, L'Organisation corporative de la France de l'Ancien Régime, Paris, 1938 ; A. L. CROSET, La France, pilote de l'Europe : des corporations du Moyen Âge à l'organisation professionnelle de l'ère nouvelle, Lyon, 1941.

25. É. DollÉAns et G. Dehove, Histoire du travail en France. Mouvement ouvrier et législation sociale, Paris, 1953 ; M. BouviER-AJAM, Les Classes sociales, Paris, 1963.

26. G. LEFRANC, Histoire du travail et des travailleurs, Paris, 1957.

27. P. WOLFF, «Les luttes sociales dans les villes du Midi français, du XIII ${ }^{\mathrm{e}}$ au XV ${ }^{\mathrm{e}}$ siècle », Annales ESC, 2 (1947), p. 443-454 ; piste reprise ensuite dans M. MoLLAT et P. WoLFF, Ongles bleus, Jacques et Ciompi : les révolutions populaires en Europe aux XIVe et XVe siècles, Paris, 1970, puis A. STELlA, La Révolte des Ciompi : les hommes, les lieux, le travail, Paris, 1993 et, plus récemment encore, M. BoONE, «Armes, coursses, assemblees et commocions : les gens de métiers et l'usage de la violence dans la société urbaine flamande à la fin du Moyen Âge », Revue du Nord, 87/359 (2005), p. 7-33.

28. B. GEREMEK, Le Salariat de l'artisanat parisien aux XIII -XVe siècles : étude sur le marché de la main-d'œuvre au Moyen Âge, Paris/La Haye/New York, 1968. Cette publication en français est postérieure de six années à l'ouvrage paru en polonais en 1962, mais c'est par elle que les chercheurs français accèdent aux travaux de Bronislaw Geremek. 
L'éclipse est plus générale encore dans les années 1970 et 1980, en dépit de quelques notables exceptions ${ }^{29}$. En dehors de la réflexion sur la notion même de travail initiée par Jacques Le Goff ${ }^{30}$, la focale se déplace sur le commerce et les échanges, et l'histoire des salaires est combinée à celle des prix $^{31}$. La recherche en histoire urbaine s'étoffant, la question du travail est désormais intégrée aux monographies de villes, en vogue à l'époque ${ }^{32}$. Les deux volumes de la Nouvelle Histoire de Paris consacrés au Moyen Âge par Raymond Cazelles et Jean Favier en 1972 et 1974 font eux aussi une place à l'artisanat ${ }^{33}$. Dans tous sont évoqués les métiers, et l'étalon parisien, même fortement nuancé, y sert encore de référence implicite tant il a forgé le cadre d'analyse de la réflexion sur l'organisation de la production. Mais d'autres approches émergent, en particulier spatiales : sont notamment examinés les lieux du travail, leur place dans la topographie des villes.

Les années 1990 marquent le renouveau de la question. En 1991, Michel Hébert constatait : « on a assez peu étudié les questions relatives au travail et à l'artisanat urbain depuis plusieurs décennies » et concluait : « Il n'est donc pas inopportun de reprendre l'enquête ${ }^{34}$ ». Dix ans plus tard, Anne Jollet partait du même «constat de la faiblesse de la curiosité des historiens », toutes périodes confondues, d'une « relative indifférence », mais pour le nuancer aussitôt en pointant, derrière une atonie apparente - largement imputable à l'absence de nouvelle grande synthèse ${ }^{35}$-, la reformulation des questions et le déplacement des centres d'intérêt en réalité déjà à l'œuvre ${ }^{36}$. La recherche remet en effet en cause certaines idées qui prévalaient dans l'historiographie passée et emprunte désormais d'autres pistes, qu'il faut se résoudre à n'évoquer que succinctement ici. Alors

29. Parmi lesquelles G. H. AlLARD et S. LuSIGNAN éd., Les Arts mécaniques au Moyen Âge, Montréal/Paris, 1982, et P. BENoîT et D. CAILlauX éd., Hommes et travail dans les villes médiévales, Actes de la table ronde La Métallurgie urbaine dans la France médiévale, Paris 23 mars 1984, Paris, 1988.

30. En particulier J. LE GofF, Pour un autre Moyen Âge. Temps, travail et culture en Occident, Paris, 1977.

31. Le meilleur exemple en est C. DE LA RONCIÈRE, Prix et salaires à Florence au XIV siècle, Rome, 1982.

32. Par exemple P. DESPORTES, Reims et les Rémois aux XIII et XIVe siècles, Paris, 1979; R. Favreau, La Ville de Poitiers à la fin du Moyen Âge, Poitiers, 1978 ; B. CheValier, Tours, ville royale (1356-1520) : origine et développement d'une capitale à la fin du Moyen Âge, Paris, 1975.

33. R. CAZELles, De la fin du règne de Philippe Auguste à Charles V, Paris, 1972 ; J. FAVIER, Paris au XV siècle, Paris, 1974.

34. M. HÉBERT, « Travail et vie urbaine à Manosque à la fin du Moyen Âge », dans C. Dolan éd., Travail et travailleurs en Europe au Moyen Âge et aux débuts des temps modernes, Toronto, 1991, p. 147-173.

35. Celle de Robert Fossier ne fait qu'une maigre place au travail urbain en raison des champs de recherche de l'auteur : R. FossIER, Le Travail au Moyen Âge, Paris, 2000.

36. A. JOLleT, « Comment les historiens parlent-ils du travail ? », Cahiers d'Histoire. Revue d'histoire critique, 83 (2001), p. 7-12. 
que le métier avait été la voie d'accès privilégiée à l'histoire du travail, il apparaît désormais comme un écran, qui masque la réalité davantage qu'il ne l'éclaire. Au mieux, le métier est désormais considéré comme un mode, parmi d'autres, de l'organisation artisanale, et pas celui qui reflète le mieux les situations réelles ; parfois même, il est regardé comme une construction artificielle, sans rapport avec la réalité ${ }^{37}$. C'est que les sources normatives sont reconsidérées au filtre d'une critique plus poussée qui amène à discuter ce que l'on peut véritablement en tirer, y compris de certains « monuments » - jusqu'au Livre des métiers d'Étienne Boileau ! - utilisés jusque-là sans suffisamment de recul. Jean-Pierre Sosson résume la position désormais commune : «Faute d'une intégration systématique des acquis de l'histoire économique et sociale et d'une utilisation des matériaux prosopographiques disponibles, les textes, pour l'essentiel de nature normative, ne peuvent donner que des images statiques, désincarnées sinon lénifiantes des métiers ${ }^{38}$. » Le modèle parisien est réfuté ${ }^{39}$ ou en tout cas nuancé dès lors que les études de cas montrent qu'il ne s'applique ni à toutes les régions, ni à tous les métiers d'un même lieu, ni à tous les travailleurs (non qualifiés, femmes, enfants, esclaves éventuels). La distinction traditionnelle entre métiers réglés et métiers jurés est jugée plus formelle que probante ${ }^{40}$. L'approche institutionnelle est de manière générale contestée, car jugée trop rigide. Une attention plus fine à la chronologie révèle une situation mouvante, loin du tableau figé dressé autrefois à partir du seul XIII ${ }^{\mathrm{e}}$ siècle ${ }^{41}$.

La méfiance par rapport à la structure corporative s'étendant aux textes réglementaires, des sources nouvelles sont sollicitées, autant fiscales, que judiciaires, notariales, littéraires ou iconographiques, susceptibles de laisser percevoir davantage d'aspects que les sources réglementaires et de donner mieux accès à la réalité. Surtout, elles sont systématiquement croisées, les unes venant en contrepoint des autres pour cerner un même objet. À ces documents sont appliquées des méthodes mises au point dans

37. « La rigidité du schéma corporatif se heurte à la multiplicité ou à la superposition des liens unissant les individus au sein de la cellule de production » : C. VERNA et P. BERNARDI, «Travail et Moyen Âge... »,p. 40.

38. J.-P. Sosson, «Les métiers : normes et réalité. L'exemple des anciens Pays-Bas méridionaux aux XIV et $\mathrm{XV}^{\mathrm{e}}$ siècles », dans J. HAMESSE et C. MURAILLE-SAMARAN éd., Le Travail au Moyen Âge. Une approche interdisciplinaire, Louvain-la-Neuve, 1990, p. 338-348 (p. 339-340).

39. Une partie du n ${ }^{\circ} 2$ des Annales HSS, 60 (2005), porte de manière très explicite en titre « Le travail sous l'Ancien Régime. Pour en finir avec le modèle standard », entendons celui transposé de Paris et hérité de l'historiographie ancienne.

40. Par exemple, M. HÉBERT, « Travail et vie urbaine à Manosque... ».

41. Michel HÉBERT écrit : «Il semble que si l'on veut réellement comprendre les origines et la structure des métiers en Provence avant le $\mathrm{XVI}^{\mathrm{e}}$ siècle, il faille définitivement se départir d'une approche trop rigidement institutionnelle. Toutes les catégories inventées surtout par les des historiens du droit, corporation, métier juré, métier libre, ne semblent guère applicables avant la fin du $X V^{\mathrm{e}}$ siècle » (ibid., p. 147). 
d'autres champs de la recherche historique, notamment la prosopographie grâce à laquelle on suit un groupe de manière très fine et dans toute la complexité de son existence, voire la micro-analyse (réhabilitée après une période marquée par l'histoire quantitative) qui fait sa place à l'individu. Est désormais privilégiée l'étude interdisciplinaire ainsi que l'annonce le colloque tenu à Louvain-la-Neuve en 1987, Le Travail au Moyen Âge. Une approche interdisciplinaire ${ }^{42}$. Les historiens nourrissent leur réflexion auprès d'autres disciplines comme la sociologie ${ }^{43}$, l'anthropologie et plus encore l'archéologie. Cette dernière, en apportant une documentation radicalement différente, fait faire de grands progrès. Elle rencontre les préoccupations d'historiens marqués par l'histoire de la civilisation matérielle. L'histoire des techniques connaît alors un développement considérable ${ }^{44}$, balisant un champ de recherche qui s'avère extrêmement fécond et produit des avancées majeures pour ce qui est de l'histoire du travail, axée davantage sur la matérialité, les outils, mais aussi sur la dimension humaine : l'homme au travail en tant qu'individu, ses gestes, son corps, ses souffrances, et ses relations de travail ${ }^{45}$.

D'un autre côté, l'histoire du genre, impulsée dans le monde anglophone, amène l'école historique française à considérer différemment la question du travail des femmes ${ }^{46}$. Celui des enfants est à son tour examiné ${ }^{47}$, ce qui réintroduit les problèmes de la situation de l'apprenti, de l'apprentissage, mais aussi de la transmission des savoirs ${ }^{48}$. Ce dernier

42. J. Hamesse et C. Muraille-Samaran éd., Le Travail au Moyen Âge...

43. Notamment les travaux de Robert Castel, dont R. CASTEL, Les Métamorphoses de la question sociale : une chronique du salariat, Paris, 1995.

44. P. BRAunstein, «Pour une historie économique et sociale des techniques », dans Des sciences et des techniques : un débat, R. GUESNERIE et F. HARTOG éd., Cahiers des Annales, 45 (1998), p. 209-217.

45. Parmi d'autres, M. ARNOUX, Mineurs, férons et maîtres de forges. Études sur la production de fer dans la Normandie du Moyen Âge, XI $-X V^{e}$ siècles, Paris, 1993 ; C. VERNA, Le Temps des moulines. Fer, technique et société dans les Pyrénées centrales (XIII ${ }^{e}$-XVI siècles), Paris, 2001.

46. Parmi une abondance d'articles, C. BÉGHIN, « Donneuses d'ouvrages, apprenties et salariées aux XIV et $\mathrm{XV}^{\mathrm{e}}$ siècles dans les sociétés urbaines languedociennes », Clio. Histoire. Femmes et société, 3 (1996), p. 31-54 ; P. BERNARDI, « Pour une étude du rôle des femmes dans le bâtiment au Moyen Âge », Provence historique, 43/173 (1993), p. 267-278 ; une synthèse : F. BAtTAGliola, Histoire du travail des femmes, Paris, 2000.

47. F. FRANCESCHI, « Les enfants au travail dans l'industrie textile florentine des XIV et $X^{\mathrm{e}}$ siècles », et K. SIMON-MUSCHEID, «Indispensable et caché. Le travail des enfants au bas Moyen Âge et à la Renaissance », dans Les Dépendances au travail, Médiévales, 30 (1996), respectivement p. 69-82 et p. 97-107.

48. Actes du $1^{\mathrm{er}}$ colloque du CRISIMA : Apprentissages, éducation, initiation, éducation au Moyen Âge, Montpellier 1991, Les cahiers du CRISIMA, 1 (1993), notamment P. BERNARDI, «Apprentissage et transmission du savoir dans les métiers du bâtiment à Aixen-Provence à la fin du Moyen Âge (1400-1550)», p. 69-79; La trasmissione dei saperi nel medioevo (secoli XII-XV), Pistoia, 2005. 
thème amène à une interrogation sur l'innovation, spécialement au sens technique, très en vogue aujourd'hui ${ }^{49}$.

Des pistes déjà abordées sont poursuivies, telle la réflexion sur la notion de travail et la valeur accordée au travail, y compris manuel ou « mécanique », celle sur la qualification d'industrie ou proto-industrie appliquée à l'artisanat médiéval ${ }^{50}$, celle enfin de l'identité professionnelle et de la place du métier dans la désignation des individus ${ }^{51}$. L'intérêt porté depuis longtemps à la production drapière demeure, et la connaissance en est renouvelée par l'histoire des techniques ${ }^{52}$. Passe cependant au premier plan l'étude des métiers de la construction, qui donne lieu à de nombreuses publications importantes ${ }^{53}$. Le métier n'apparaissant plus comme un cadre d'analyse satisfaisant, le regard se porte sur d'autres formes d'organisation du travail qui apparaissent comme au moins complémentaires sinon plus pertinentes : l'atelier, le chantier ${ }^{54}$, la filière. L'artisanat n'est plus cantonné au cadre étroit d'une ville mais analysé au sein d'ensembles plus vastes : la ville et ses périphéries, la région voire l'espace interrégional ${ }^{55}$. Une démarche nouvelle et porteuse de résultats très prometteurs consiste à

49. Notamment D. MEEKS et D. GARCIA éd., Techniques et économie antiques et médiévales : le temps de l'innovation (colloque international Aix-en-Provence, 21-23 mai 1996), Paris, 1997 ; P. BECK éd., L'Innovation technique au Moyen Âge, Actes du VI congrès international d'archéologie médiévale (1-5 octobre 1996, Dijon/Mont Beuvray/Chenôve/Le Creusot/Montbard), Caen/Paris, 1998 ; Techniques : les paris de l'innovation, Médiévales, 39 (2000) ; M. BALARD et M. SOT éd., Au Moyen Âge, entre tradition antique et innovation, Actes du $131^{\mathrm{e}}$ congrès national des sociétés historiques et scientifiques, Grenoble, 24-29 octobre 2006, Paris, 2009.

50. P. BRAUNSTEIN, «L'industrie à la fin du Moyen Âge : un objet historique nouveau?», dans L. BERGERON et P. BOURDELAIS éd., La France n'est-elle pas douée pour l'industrie?, Paris, 1998, p. 25-40.

51. P. BERNARDI, «Le métier : réflexions sur un mode d'identification », dans M. ARNOUX et P. MONNET éd., Le Technicien dans la cité..., p. 93-107.

52. D. CARDon, La Draperie au Moyen Âge. Essor d'une grande industrie européenne, Paris, 1999.

53. Parmi lesquelles J. TARDIEU et N. REVEYRON éd., L'Échafaudage dans le chantier médiéval, Lyon, 1996 ; P. LARDIN, Les Chantiers du bâtiment en Normandie orientale (XIVeXVI $I^{e}$ siècles). Les matériaux et les hommes, Villeneuve-d'Ascq, 2001.

54. M. PHILIPPE, «Chantier ou atelier : aspects de la verrerie normande aux XIV et $\mathrm{XV}^{\mathrm{e}}$ siècles », Annales de Normandie, 42/3 (1992), p. 239-257.

55. D. Clauzel et S. CALONNE, « Artisanat rural et marché urbain : la draperie à Lille et dans ses campagnes à la fin du Moyen Âge », Revue du Nord, 72 (1990), p. 553-554; M. ARnOuX et J. BotTIN, «Les acteurs d'un processus industriel. Drapiers et ouvriers de la draperie entre Rouen et Paris (XIV'- $\mathrm{XVI}^{\mathrm{e}}$ siècle) », dans M. ARNOUX et P. MONNET éd., Le Technicien dans la cité..., p. 347-380. 
mener l'étude à partir d'un produit : fer ou métal en général ${ }^{56}$, pierre ${ }^{57}$, laine, verre ${ }^{58}$, céramique ${ }^{59}$, etc.

On le constate : il s'agit bien d'une « effervescence » qui se lit dans les nombreux colloques, tables rondes et autres séminaires tenus sur le sujet ${ }^{60}$, occasions pour les historiens de fructueux échanges, et dont la publication fournit un matériel aussi précieux que fractionné, qu'on espère prélude à de futures synthèses. Dans ce tableau d'ensemble, la place de Paris semble faible, ce qui s'explique par le rejet dont fait l'objet l'archétype des métiers parisiens. Les recherches actuelles sur Paris s'inscrivent pourtant dans le mouvement général et ont profité des études menées sur d'autres espaces qui ont en quelque sorte tendu un miroir à la capitale. Sur le plan des méthodes, les sources normatives parisiennes ont été soumises à une critique plus rigoureuse. Les sources fiscales, notamment les rôles de taille, même si elles n'ont pas la précision des documents italiens, ont été aussi mises à contribution après une soigneuse analyse ${ }^{61}$. D'autres sources encore, autant textuelles ${ }^{62}$ qu'archéologiques, sont mobilisées et confrontées les unes aux autres. Les thématiques sont à l'unisson de la recherche. Le travail des Parisiennes a ainsi fait l'objet de nombreuses recherches ${ }^{63}$, de même

56. P. Benoit et O. Chapelot éd., Pierre et métal dans le bâtiment au Moyen Âge, Paris, 1985.

57. J. LORENZ, D. COULON et G. TARDY éd., La Pierre dans la ville antique et médiévale : analyse, méthodes et apports, Saint-Marcel, 2000.

58. S. LAGABRIELLE, " La verrerie du XII ${ }^{\mathrm{e}}$ à la fin du XV siècle : évolution d'une technique », Médiévales, 39 (2000), p. 57-78.

59. Démarche déjà suivie par La Céramique (Ve-XIXe siècle) : fabrication, commercialisation, utilisation, Actes du premier congrès international d'archéologie médiévale (Paris, 4-6 octobre 1985), Caen, 1987, p. 139-147 : plus récemment, G. PAGÈs, Artisanat et économie du fer en France méditerranéenne de l'Antiquité au début du Moyen-Âge : une approche interdisciplinaire, Montagnac, 2010.

60. Outre ceux déjà cités, il convient de mentionner P. LAMBRECHTS et J.-P. SOSSON éd., Les Métiers au Moyen Âge, aspects économiques et sociaux, Actes du colloque international de Louvain-la-Neuve, 7-9 octobre 1993, Louvain-la-Neuve, 1994 ; N. COQUERY, L. HILAIREPérez, L. SAllmann et C. Vernaéd., Artisans, industrie. Nouvelles révolutions du Moyen Âge à nos jours, Lyon, 2004.

61. L. FossIER, L'Artisanat parisien à la fin du XIII' siècle d'après les rôles de taille : critique d'une source, Rome, 1988 ; dans un cas particulier : P. MILLE, «Les métiers du bois à Paris dans les registres des tailles de la fin du XIII ${ }^{e}$ siècle et du début du XIV viècle », Paris et Île-de-France. Mémoires, 60 (2009), p. 53-81; et à titre de comparaison : M. SCHERMAN, Familles et travail à Trévise à la fin du Moyen Âge, vers 1434-vers 1509, Rome, 2013.

62. Le Dictionarius de Jean de Garlande a été réexaminé par F. LACHAUD, « La première description des métiers de Paris : le Dictionarius de Jean de Garlande (vers 1220-1230) », Histoire urbaine, 16 (2006), p. 91-114.

63. S. RouX, «Les femmes dans les métiers parisiens, XIII $-\mathrm{XV}^{\mathrm{e}}$ siècle », Clio. Histoire. Femmes et société, 3 (1996), p. 13-30 ; S. FARMER, « Down and Out and Female in ThirteenthCentury Paris », American Review, 103/2 (1998), p. 344-372 ; K. FIANU, « Les femmes dans les métiers du livre à Paris (XIII $-\mathrm{XIV}^{\mathrm{e}}$ siècle) », dans H. SPILLING éd., La Collaboration 
que la construction ${ }^{64}$. L'étude des techniques et des matériaux n'est pas en reste ${ }^{65}$. Paris conserve malgré tout quelques spécificités parmi lesquelles la place faite à l'artisanat du livre ${ }^{66}$.

À cela, il faut ajouter les articles de ce volume qui, chacun à sa manière, reflètent aussi les tendances actuelles de la recherche, appliquée à la capitale et à ses abords.

Caroline Bourlet reprend le dossier de la réglementation du travail dans la capitale à partir d'une critique renouvelée de ces textes, s'attachant à en prendre l'exacte mesure, à les replacer aussi dans leur contexte et à les suivre dans leur chronologie. Marion Bernard, dépassant les études par métier, s'intéresse à un groupe de professionnels aux activités voisines (même si l'on peut difficilement parler ici d'une filière) et confronte les sources réglementaires à d'autres pour tenter de démêler image et réalité du métier, en plus de s'efforcer d'atteindre les travailleurs eux-mêmes par le recours à la prosopographie. L'article de Patrick Rambourg éclaire quant à lui un secteur rarement étudié mais combien présent dans les villes en général, et dans Paris en particulier, celui des métiers de bouche. Sharon Farmer et Boris Bove traitent des activités du textile, mais à travers des aspects particuliers et sous deux angles différents : alors que Sharon Farmer se penche sur une branche spécifique de la production, celle de la soie, à Paris, Boris Bove examine la question d'un espace régional de la draperie à travers un cas de conflit relatif à la teinturerie à Saint-Denis. Le parti a en effet été pris d'étendre le terrain d'investigation en retenant ici des contributions portant sur cette localité voisine, considérée dès les origines comme un satellite de Paris et étudiée comme telle par les historiens, y compris au

dans la production de l'écrit médiéval, Actes du XIII colloque du Comité international de paléographie latine (Weingarten, septembre 2000), Paris, 2003.

64. Après la voie ouverte par les travaux de Jean Chapelot sur le château de Vincennes (notamment Le Château de Vincennes : une résidence royale au Moyen Âge, Paris, 1994), Yvan Lafarge a soutenu en 2013 une thèse sur « Le plâtre dans la construction en Île-deFrance : techniques, morphologie et économie avant l'industrialisation », sous la direction du professeur Anne-Françoise Garçon, à l'Université Paris 1 Panthéon-Sorbonne.

65. E. NeChtine, «Les artisans du métal à Paris, $\mathrm{XIII}^{\mathrm{e}}-\mathrm{XV}^{\mathrm{e}}$ siècles », dans P. BENOÎT et D. CAILlaux, Hommes et travail..., p. 29-60.

66. K. FIANU, «Familles et solidarités dans les métiers du livre parisien au XIV ${ }^{\mathrm{e}}$ siècle », Médiévales, 19 (1990), p. 83-90 ; EAD., « Les professionnels du livre à la fin du XIII ${ }^{\mathrm{e}}$ siècle : l'enseignement des registres fiscaux parisiens », Bibliothèque de l'École des chartes, 150/2 (1992), p. 185-222 ; R. H. et M. A. Rouse, Manuscripts and Their Makers : Commercial Book Producers in Medieval Paris, 1200-1500, Turnhout, 2000 ; G. CROENEN et P. AINSWORTH, Patrons, Authors and Workshops : Books and Book Production in Paris around 1400, Louvain/Paris/Dudley (Mass.), 2006 ; F. BARBIER éd., Paris capitale des livres. Le monde des livres et de la presse à Paris, du Moyen Âge au XXe siècle, Paris, 2007 (notamment le chapitre 1: «Du manuscrit à l'imprimé, les derniers siècles du Moyen Âge »). 
plan économique ${ }^{67}$. Cette étroite dépendance a privé la ville de Saint-Denis d'une historiographie propre et l'histoire de l'artisanat y reste largement à faire. En outre, le cas de Saint-Denis permet d'aborder le système productif parisien du point de vue de son intégration à l'échelle d'une région, en interrogeant les relations de complémentarité, d'influence, de concurrence et de domination de la ville-centre sur ses périphéries. L'abbaye de SaintDenis fournit aussi l'occasion à Maxime L'Héritier d'étudier le chantier comme modèle d'organisation du travail distinct de l'atelier, et lève le voile sur la situation de la construction à proximité immédiate de la capitale.

Tous ensemble, les travaux présentés témoignent de l'activité de la recherche sur le travail à Paris à la fin du Moyen Âge.

Christine Jéhanno - Université du Littoral Côte d'Opale

67. À preuve le fait que la draperie sandyonisienne apparaît dans R. GouRMELON, L'Industrie et le commerce des draps à Paris du XIII a a XVI siècle, thèse de l'École des chartes, 1950, 2 vol., où elle est étudiée pour sa complémentarité avec celle de la capitale. 
